\title{
Improving the Accuracy of Estimates of the Pulse Sequence Period Using the Methodology of Complete Sufficient Statistics
}

Константин Жучков ( $\square$ konst_z@outlook.com )

Gubkin Russian State University of Oil and Gas

Mikhail Vasilchenko

RMIT University

Anna Zagrebneva

Don State Technical University

Alexey Zavyalov

Gubkin Russian State University of Oil and Gas

\section{Research Article}

Keywords: period estimation, quasi-rectangular pulses, complete sufficient statistics, maximum likelihood estimation

Posted Date: March 1st, 2022

DOI: https://doi.org/10.21203/rs.3.rs-1388683/v1

License: (9) This work is licensed under a Creative Commons Attribution 4.0 International License. Read Full License 


\title{
Improving the accuracy of estimates of the pulse sequence period using the methodology of complete sufficient statistics
}

Zhuchkov $\mathrm{K}^{\mathrm{a}}$, Vasilchenko $\mathrm{M}^{\mathrm{b}}$., Zagrebneva $\mathrm{A}^{\mathrm{c}}$, Zavyalov $\mathrm{A}^{\mathrm{d}}$.

a - National University of Oil and Gas “Gubkin University”, 65, bld. 1 Leninsky prospekt., Moscow, 119991, Russia (e-mail: konst_z@outlook.com).

b - School of Engineering, RMIT University, GPO Box 2476, Melbourne VIC 3001, Australia (e-mail: s3661446@student.rmit.edu.au)

c - Faculty of IT Systems and Technologies, Don State Technical University, Gagarin sqri, Rostov-on-Don, 344000, Russia (anna.zagrebneva@gmail.com)

d - National University of Oil and Gas “Gubkin University”, 65, bld. 1 Leninsky prospekt., Moscow, 119991, Russia (e-mail: zavyalovap@yandex.ru ).

\begin{abstract}
Using a model of a sequence of quasi-rectangular pulses as an example, an approach was illustrated to assess their period, taking into account the off-duty factor and the squareness coefficient of the pulses. Complete sufficient statistics (CSS) methodology was used. An analytical expression for the pulse repetition was presented. It was shown that for the value of the sample size ( $\mathrm{n}>8$ ) the relative root-mean square error of the repetition period estimation by CSS is shown as a significantly smaller error than that of the traditional estimate, which is only asymptotically effective. In addition to theoretical calculations, simulation results confirming the achieved effect are presented. The results obtained are applicable for the design of the control and measuring equipment of the oil and gas industry.
\end{abstract}

Keywords_-period estimation, quasi-rectangular pulses, complete sufficient statistics, maximum likelihood estimation.

\section{Introduction}

The task of the parameter precision estimation for the periodic and quasi-periodic signals of varying natures has not lost its relevance for several decades. The traditional field of application for devices that solve such problems are: radars [1], communication systems [2], [3] and electronic intelligence [4]. However, the range of applications much wider and includes, for example, the assessment of the parameters of X-Ray absorption spectra [5], Bragg scattering [6], plasma parameters [7,35], assessment of the stability of the information system [8], the solution of the problem of the parameter estimation for in-line flaw detection [9], or the development of an optoelectronic displacement sensors [10], which are used in the oil and gas industry.

In the condition of low a priori certainty in the initial data, attempts are made to solve the problem of evaluating the efficiency of the algorithm for measuring signal parameters against the background of noise using the classical Bayesian criterion [11]. However, the complexity of these attempts is associated with the requirement to a priori probabilities of the presence and absence of a signal in the observed sample, the distribution functions of probabilities and losses.

In the absence of a priori information on both the magnitude of losses and the probabilities of the presence and absence of a signal in the original sample the maximum likelihood criterion is used [12]. It is assumed that the loss function is a constant, and the structure of the algorithms in this case depends on the vector of interfering parameters. As applied to the problems of estimating signal parameters the maximum likelihood method turned out to be the most effective: unknown interfering parameters are included in the estimated parameters and estimates are found for all parameters, including those that are insignificant for the given problem [13].

At the same time, it is well known [12] that under conditions of the Gaussian noise maximum likelihood estimation (MLE) is asymptotically optimal, i.e., the stability of its operations and the efficiency of the estimates are achieved at high signal/noise ratios and an infinite sample size. In the context of the problems of estimating the parameters of real signals, there is a need to search for algorithms that can work no worse with finite samples and low signal/noise ratios. That way, for example, in order to perform the parameter estimation for the sine combination signals and periodic signals the multi-innovation stochastic gradient algorithm based on the gradient optimization principle is presented in [14]. A closed-form maximum likelihood amplitude estimator is obtained in [15]. The extended noise-resistant correlation method to identify the period of pseudoperiodic signals is derived in [16]. Attempts are being made to use Fourier and wavelet transformation with windows of various kinds to increase the localization of signal energy [17], [18] or multithreshold circuits [19], [20] are used, which give a gain in the accuracy of the estimate in particular cases, however, in general, they carry the risk of estimation circuit work going from stable and equilibrium state. It is also possible to use neural network methods to solve the problem of estimating signal parameters, but the main disadvantage here is multiple increase in the sample size for training [21], even with a slight change to the signalnoise environment.

Methods for synthesizing estimation algorithms include methods of complete sufficient statistics based on the use of the Rao-Blackwell estimate for Lehmann-Scheffe theorem [22], [23]. If the conditioning statistic is complete and sufficient and the initial estimate is unbiased, then the Rao-Blackwell estimate is the single best unbiased estimate [24].

In radio engineering applications, the distributions of samples and observed technical processes often have complete sufficient statistics, which creates the prerequisites for the application of the Lehmann-Scheffe theorem in finding effective estimates of signal parameters. This paper shows the application of CSS methods to find an effective estimate of the pulse repetition period against the background of noise and interference under conditions of parametric a priori uncertainty. The application of the estimates obtained can find a place not only in traditional problems of estimating the repetition period, but also in several problems that reduce the problem of finding the repetition period. For example, when implementing clock recovery circuit for a demodulator operating on short signal samples [25], when the phase-locked loop capture time is significantly longer than the signal sample duration [26].

\section{Mathematical signal model}

Consider the process $x(t)$ on the segment $[0, \Delta T]$, which is an additive mixture of signal and noise: 


$$
x(t)=A_{m} s(t)+\eta(t) .
$$

where $A_{m}$ is the signal amplitude, $s(t)$ is the normalized signal, $\eta(t)$ is the differentiable stationary Gaussian noise characterized by the variance $\sigma^{2}$ and the normalized correlation function $r(t)$. The ration of the energy parameters of the signal and noise will be characterized by the signal/noise ratio $q=A_{m} / \sigma$.

Let the leading edges of the signal pulse $s(t)$ intersect the level $H$ at time $t_{0 i}^{+}$, and the trailing at time $t_{0 i}^{-}$:

$$
\begin{aligned}
& t_{0 i}^{+}=t_{00}^{+}+i T, \quad i=0,1, \ldots \\
& t_{0 i}^{-}=t_{00}^{+}+i T+T / v, \quad i=0,1, \ldots,
\end{aligned}
$$

where $T$ is the signal period; $v$ is the off-duty factor; $t_{00}^{+}$is the first crossing of $H$ level. Then, for large signal/noise ratios, $\mathrm{q}>>1$, the process $x(t)$ will cross the $H$ level at times

$$
t_{i}^{+}=t_{0 i}^{+}+\Delta t_{0 i}^{+}, \quad t_{i}^{-}=t_{0 i}^{-}+\Delta t_{0 i}^{-},
$$

where $\Delta t_{0 i}^{+}, \Delta t_{0 i}^{-}$are small random additions, due to effect of noise $\eta(t)$. Fig. 1 schematically shows the process and the designations adopted in the work.

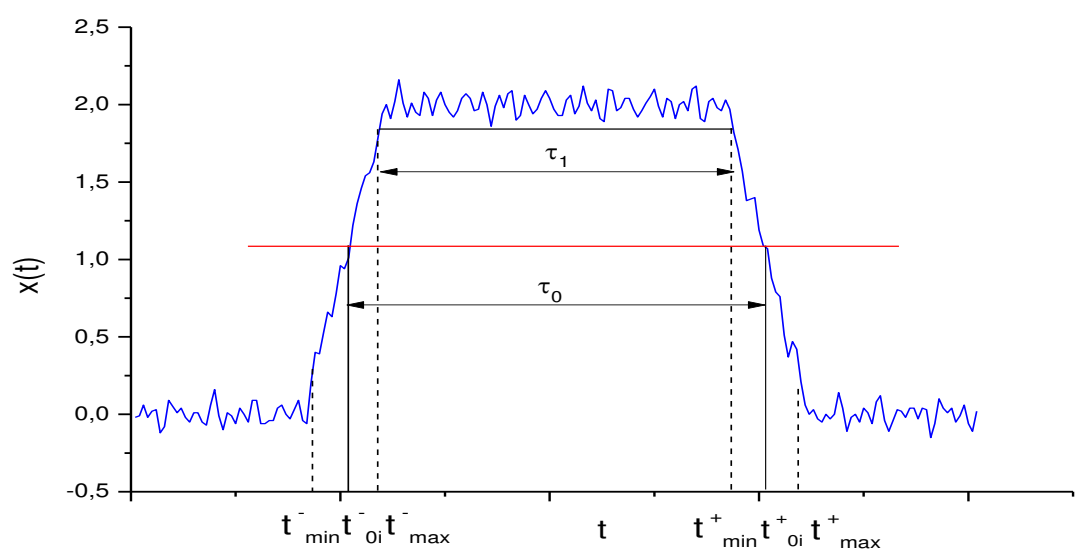

Fig.1. Observed process $x(t)$ in the form of the impulse on the noise background.

It is assumed that the pulses have a quasi-rectangular shape and can be approximated according to [27] by the following function

$$
s(x)= \begin{cases}\exp \left\{-\frac{\pi}{8} \frac{(2 x k-1)^{2}}{(k-1)^{2}}\right\}, & x>\frac{1}{2 k}, \\ 1, \quad|x| \leq \frac{1}{2 k}, & \\ \exp \left\{-\frac{\pi}{8} \frac{(2 x k+1)^{2}}{(k-1)^{2}}\right\}, & x<-\frac{1}{2 k} .\end{cases}
$$

Here $k$ is the coefficient of the pulse squareness, it equals the ratio of pulse duration at the intersection of $H$ level $\tau_{0}$ to the pulse duration $\tau_{1}, k=\tau_{0} / \tau_{1}$ (see Fig. 1).

The objective of this research is to synthesize the most effective estimation of the repetition period of quasi-rectangular pulses against the background noise and interference in the conditions of a priori uncertainty its repeatability (period and phase). An additional complication of the model is the possible loss of a pulse in the sequence according to a pseudorandom law, which is not known a priori. The known parameters are: off-duty factor (excluding loss), squareness coefficient, the times when the signal leading and trailing edges cross the $\mathrm{H}$ level.

\section{Methodology}

\subsection{Description of maximum likelihood estimation method.}


The measurement process of the pulse repetition period is derived from the process of measuring the time of the beginning (end) of the pulse. Taking into account that the parameter (classical in the theory of maximum likelihood estimates - MLE) is non-energetic, in estimating the lower bound of the accuracy of pulse repetition period measurement, we take as a basis the expression for the elements of the Fisher matrix:

$$
\Phi_{i k}=-\left.q^{2} \frac{\partial^{2} \Psi(\lambda)}{\partial \lambda_{i} \partial \lambda_{k}}\right|_{\lambda=0},
$$

where $q$ is the signal/noise ratio, $\Psi(\lambda)$ is the uncertainty function of the vector $\lambda$. Since the inverse Fisher matrix is the correlation matrix of the MLE of all simultaneously estimated parameters [28], and the dispersions of the MLE of these parameters are on the main diagonal of this matrix, and taking into account that the focus is only on one non-energy parameter - the pulse arrival time $(\tilde{t})$, it can be written as:

$$
D\{\hat{\tilde{t}} \mid \tilde{t}\}=-1 / q^{2} \Psi^{\prime \prime}(0)
$$

where $\hat{\tilde{t}}$ is the MLE of the pulse arrival time. Assuming that the uncertainty function is stationary with respect to the parameter $t-\tilde{t}$ it gives the following expression:

$$
\Psi(t-\tilde{t})=\frac{1}{2 E} \int_{-\infty}^{\infty} s(t-\tilde{t}) s^{*}(t) d t
$$

where $s(t)$ is the complex envelope of the signal, and $E$ is its energy. After applying Parseval theorem and double differentiation (3) dispersion of MLE for the parameter $\tilde{t}$ is bounded by the expression below:

$$
D\{\hat{\tilde{t}} \mid \tilde{t}\}=\frac{\int_{-\infty}^{\infty}|S(f)|^{2} d f}{4 \pi^{2} q^{2} \int_{-\infty}^{\infty} f^{2}|S(f)|^{2} d f},
$$

where $S(f)$ is the Fourier transformation of the complex envelop of the signal $s(t)$. Taking into account the approximation in which the pulse is rectangular [34] with duration $\tau_{0}$ (passed through a perfect filter with passband $\Delta \mathrm{f}$, where $\Delta \mathrm{f} \tau_{0} \approx 1$ ), the following assumption can be used $\left(\mathrm{S}(\mathrm{f})=\left|\sin \left(\pi \mathrm{f} \tau_{0}\right)\right| / \mathrm{f} \tau_{0}\right.$ and normalization condition for $\left.\mathrm{S}(\mathrm{f})[23]\right)$ :

$$
\frac{\int_{-\infty}^{\infty} f^{2}|S(f)|^{2} d f}{\int_{-\infty}^{\infty}|S(f)|^{2} d f} \approx \frac{1}{\tau_{0}^{2}} .
$$

In this case, if a sequence of pulses with an off-duty factor $v=T / \tau_{0}=2$ (meander) is an input of the meter, the lower boundary of the estimate variance of the arrival time estimate for each pulse considering (5) is presented as:

$$
D\{\hat{\tilde{t}} \mid \tilde{t}\}=\frac{T^{2}}{16 \pi^{2} q^{2}} .
$$

The most common method for estimating the period is based on measuring the difference between the start times of adjacent pulses. If the assessment is made by $n$ adjacent periods, then its relative error decreases $n$ times

$$
\delta_{0}=\frac{\sqrt{D\{\hat{\tilde{t}} \mid \tilde{t}\}}}{n T}=\frac{1}{4 \pi q n} .
$$

If the period is estimated using the information contained in the statistics of the pulse end times (crossing threshold by the trailing edge), then the estimate can be improved by $\sqrt{2}$. Thus, we can assume that for traditional estimates the video pulse repetition with an off duty factor $=2$, the relative root-mean-square (RMS) error is

$$
\delta_{0}=\frac{1}{4 \sqrt{2} \pi q n} .
$$

The estimate (11) is the asymptotically accuracy bound for the method (the limiting value of the Cramer-Rao inequality [12]).

An example of the traditional estimation of the pulse repetition period by the MLE method is the expression: 


$$
\tilde{T}=\frac{1}{2 n} \sum_{k=1}^{n}\left(\frac{t_{k}^{+}-t_{k-1}^{+}+t_{k}^{-}-t_{k-1}^{-}}{i_{k}-i_{k-1}}\right),
$$

where, $t_{k}^{+}, t_{k}^{-}$are the times of intersection of the leading and trailing pulse edges (10), $i_{k}$ is the number of the pulse in the sequence. When determining the numbers $i_{k}$, all pulses are considered, even those that were missed and did not fall into the sample.

\subsection{Description of complete sufficient statistics estimation method.}

Consider the statistics $\boldsymbol{t}^{+}=\left\{t_{0}^{+}, \ldots, t_{n}^{+}\right\}$and $\boldsymbol{t}^{-}=\left\{t_{0}^{-}, \ldots, t_{n}^{-}\right\}$, where $t_{i}^{+}$and $t_{i}^{-}$are given (2) to synthesize the estimate of the period $T$. To find the joint probability density of the statistics $t^{+}$and $t^{-}$, the results of [22] - [26] are used. Suppose that with a probability close to one, the crossing of the level $H$ will occur in disjointed bounds $\left(t_{i \min }^{+}, t_{i \max }^{+}\right)$and $\left(t_{i \min }^{-}, t_{i \max }^{-}\right)$points $t_{0 i}^{+}$and $t_{0 i}^{-}$(Fig. 1). If the probabilities of false crossing of the level $H$ are small and the condition below is satisfied [19]:

$$
\begin{gathered}
\Phi\left(\frac{U_{m}\left|s^{\prime}\left(t_{0 i}^{-}\right)\right|}{\sigma_{1}}\right)+\frac{\sigma_{1}}{U_{m}\left|s^{\prime}\left(t_{0 i}^{-}\right)\right| \sqrt{2 \pi}} \exp \left\{\frac{U_{m}^{2}\left|s^{\prime}\left(t_{0 i}^{-}\right)\right|^{2}}{\sigma_{1}^{2}}\right\} \approx 1, \\
\Phi(x)=\frac{1}{\sqrt{2 \pi}} \int_{-\infty}^{x} e^{-\frac{t^{2}}{2}} d t,
\end{gathered}
$$

where $\Phi(x)$ is the Laplace integral, $\sigma_{1}^{2}=-\sigma r^{\prime \prime}(0)$ is the variance of the derivative of the process $\eta(t), r^{\prime \prime}(0)$ is the value of the second derivative of the normalized correlation function $r(t)$ at $t=0$, then the one dimensional probabilities distribution densities at level $H$ looks as following [23]:

$$
\begin{aligned}
& \rho\left(t_{i}^{+}\right)=\frac{1}{\sqrt{2 \pi \sigma_{+}^{2}}} \exp \left\{-\frac{\left(t_{i}^{+}-t_{o o}^{+}-i T\right)^{2}}{2 \sigma_{+}^{2}}\right\}, \\
& \rho\left(t_{i}^{-}\right)=\frac{1}{\sqrt{2 \pi \sigma_{-}^{2}}} \exp \left\{-\frac{\left(t_{i}^{-}-t_{o o}^{+}-T / v-i T\right)^{2}}{2 \sigma_{-}^{2}}\right\},
\end{aligned}
$$

where $\sigma_{+}=1 /\left[q\left|s^{\prime}\left(t_{0 i}^{+}\right)\right|\right], \quad \sigma_{-}=1 /\left[q\left|s^{\prime}\left(t_{0 i}^{-}\right)\right|\right]$

Then, under the assumption that the processes within the intervals of the leading and trailing edges of the pulse will be statistically independent, the joint probability density distribution is obtained for the sample vectors $\mathbf{t}^{+}$и $\mathbf{t}^{-}$:

$$
\rho\left(\mathbf{t}^{+}, \mathbf{t}^{-}\right)=\prod_{i=0}^{n} \rho\left(t_{i}^{+}\right) \rho\left(t_{i}^{-}\right)=\left(\frac{1}{2 \pi \sigma_{+} \sigma_{-}}\right)^{n+1} \times \exp \left\{-\sum_{i=0}^{n} \frac{\left(t_{i}^{+}-t_{o o}^{+}-i T\right)^{2}}{2 \sigma_{+}^{2}}-\sum_{i=0}^{n} \frac{\left(t_{i}^{-}-t_{o o}^{+}-T / v-i T\right)^{2}}{2 \sigma_{-}^{2}}\right\} .
$$

Then we find an effective estimate of the pulse repetition period, assuming that the moduli of the slope of the leading and trailing edges of the signal coincide, $\left|s^{\prime}\left(t_{0 i}^{+}\right)\right|=\left|s^{\prime}\left(t_{0 i}^{-}\right)\right|$(i.e. parameters $\sigma_{+}$and $\sigma_{-}$are equal). We suppose that the off-duty factor $v$ is known, and signal noise ration $q$, the value of the modulus of the front slope $\left|s^{\prime}\left(t_{0 i}^{-}\right)\right|$, the initial signal phase is not a priori determined.

The solution of this problem using methodology of CSS is based on the theorem of the uniqueness of an effective estimate [28], which is a consequence of Lehmann-Scheffe theorem [22].

According to the uniqueness theorem for the effective estimate, if the estimated signal characteristic parameter $\mu$ can be presented as a function depending on the parameter $\mathbf{Y}=\left\{Y_{1}, Y_{2}, \ldots Y_{m}\right\}$, where $\mathbf{Y}$ is a vector of expansion of the joint probability distribution density $\rho\left(\mathbf{t}^{+}, \mathbf{t}^{-}\right)$:

$$
\mu=g(\mathbf{Y})=\sum_{i=1}^{M} \sum_{j=0}^{N} a_{i, j} m_{i}^{j}(\mathbf{Y}), \quad-\infty<a_{i, j}<+\infty .
$$

Then the only effective estimate of the parameter $\mu$ is given $[22,28]$ by the expression: 


$$
\mu^{*}=\sum_{i=1}^{M} \sum_{j=0}^{N} a_{i, j} Y_{i}^{j}
$$

Here, $M$ is the dimension of the complete sufficient statistics $\mathbf{Y}=\left\{Y_{1}, Y_{2}, \ldots Y_{m}\right\}, N$ is the maximum number of the moment order, $m_{i}^{j}(\mathbf{Y})=M\left\{Y_{i}^{j}\right\}, i=1, \ldots, M ; j=0, \ldots, N ; m_{i}^{0}(\mathbf{Y})=1$.

Let 's define complete sufficient statistics $\mathbf{Y}=\left\{Y_{1}, Y_{2}, \ldots Y_{m}\right\}$. Considering the conditions of the problem $\left(\sigma_{+}=\sigma_{-}\right)$, the joint probability distribution density $\rho\left(\mathbf{t}^{+}, \mathbf{t}^{-}\right)$(15) has the following form:

$$
\rho\left(\mathbf{t}^{+}, \mathbf{t}^{-}\right)=\left(\frac{1}{2 \pi \sigma_{+}^{2}}\right)^{n+1} \times \exp \left\{-\sum_{i=0}^{n} \frac{\left(t_{i}^{+}-t_{o o}^{+}-i T\right)^{2}}{2 \sigma_{+}^{2}}-\sum_{i=0}^{n} \frac{\left(t_{i}^{-}-t_{o o}^{+}-T / v-i T\right)^{2}}{2 \sigma_{+}^{2}}\right\} .
$$

A practical way to find sufficient statistics $\mathbf{Y}(x)$ is to use the factorization theorem [28, 32, 33], according to which the conditional probability density distribution $\rho_{\theta}(x)=P(X=x \mid \boldsymbol{\theta})$ must be represented as:

$$
\rho_{\theta}(x)=h(x) g(\boldsymbol{\theta}, \mathbf{Y}(x)) .
$$

The resulting expression for $\mathbf{Y}(x)$ is a sufficient statistic.

We factorize the conditional probability density $\rho\left(\mathbf{t}^{+}, \mathbf{t}^{-}\right)$(18) for fixed parameters $\sigma_{+}, t_{00}^{+}, T$. We denote:

$$
\boldsymbol{\theta}=\left\{\vartheta_{1}, \vartheta_{2}, \vartheta_{3}\right\}=\left\{\frac{t_{00}^{+}}{\sigma_{+}^{2}}, \frac{T}{\sigma_{+2}^{2}},-\frac{1}{\sigma_{+}^{2}}\right\} .
$$

Considering the first sum in (18) and collecting the terms containing random variables with the parameters $\vartheta_{l}, \vartheta_{2}, \vartheta_{3}$, the remaining terms are assigned to the function $C_{l}\left(\vartheta_{l}, \vartheta_{2}, \vartheta_{3}\right)$ :

$$
\begin{aligned}
& -\sum_{i=0}^{n} \frac{\left(t_{i}^{+}-t_{o o}^{+}-i T\right)^{2}}{2 \sigma_{+}^{2}}=-\frac{1}{2 \sigma_{+}^{2}} \sum_{i=0}^{n}\left(t_{i}^{+}\right)^{2}-2 t_{o o}^{+} t_{i}^{+}-2 i T t_{i}^{+}+2 i T t_{o o}^{+}+(i T)^{2}+\left(t_{o o}^{+}\right)^{2}= \\
& =-\frac{1}{2 \sigma_{+}^{2}} \sum_{i=0}^{n}\left(t_{i}^{+}\right)^{2}+\frac{t_{o o}^{+}}{\sigma_{+}^{2}} \sum_{i=0}^{n} t_{i}^{+}+\frac{T}{\sigma_{+}^{2}} \sum_{i=0}^{n} t_{i}^{+} i-\frac{t_{o o}^{+}}{\sigma_{+}^{2}} T \frac{n(n+1)}{2}-\frac{\left(t_{o o}^{+}\right)^{2}(n+1)}{2 \sigma_{+}^{2}}-\frac{T^{2}}{2 \sigma_{+}^{2}} \frac{n(n+1)(2 n+1)}{6}= \\
& =\vartheta_{1} \sum_{i=0}^{n} t_{i}^{+}+\vartheta_{2} \sum_{i=0}^{n} t_{i}^{+} i+\vartheta_{3} \sum_{i=0}^{n}\left(t_{i}^{+}\right)^{2}+C_{1}\left(\vartheta_{1}, \vartheta_{2}, \vartheta_{3}\right), \\
& C_{1}\left(\vartheta_{1}, \vartheta_{2}, \vartheta_{3}\right)=\frac{(n+1) \vartheta_{1}^{2}}{4 \vartheta_{3}}+\frac{n(n+1) \vartheta_{1} \vartheta_{2}}{2 \vartheta_{3}}+\frac{\vartheta_{2}^{2}}{4 \vartheta_{3}} \frac{n(n+1)(2 n+1)}{6}
\end{aligned}
$$

Similarly, an expression for another sum (18) gets:

$$
\begin{gathered}
-\sum_{i=0}^{n} \frac{\left(t_{i}^{+}-t_{o o}^{+}-i T\right)^{2}}{2 \sigma_{+}^{2}}=\vartheta_{1} \sum_{i=0}^{n} t_{i}^{-}+\vartheta_{2} \sum_{i=0}^{n} t_{i}^{-}(i+1 / v)+\vartheta_{3} \sum_{i=0}^{n}\left(t_{i}^{-}\right)^{2}+C_{2}\left(\vartheta_{1}, \vartheta_{2}, \vartheta_{3}\right), \\
C_{2}\left(\vartheta_{1}, \vartheta_{2}, \vartheta_{3}\right)=\frac{(n+1) \vartheta_{1}^{2}}{4 \vartheta_{3}}+\frac{(n+1) \vartheta_{1} \vartheta_{2}}{2 v \vartheta_{3}}+\frac{\vartheta_{2}^{2}}{4 \vartheta_{3}} \frac{(n+1)(n v+1)}{v^{2}}
\end{gathered}
$$

After factorization, the probability density distribution $\rho\left(\mathbf{t}^{+}, \mathbf{t}^{-}\right)$(18), taking into account (19) and the transformations performed above, takes the form

$$
\rho\left(\mathbf{t}^{+}, \mathbf{t}^{-}\right)=C(\boldsymbol{\theta}) \exp \left\{\vartheta_{1} Y_{1}+\vartheta_{2} Y_{2}+\vartheta_{3} Y_{3}\right\}
$$

where 


$$
\begin{gathered}
Y_{1}=\sum_{i=0}^{n}\left(t_{i}^{+}+t_{i}^{-}\right) ; \quad Y_{2}=\sum_{i=0}^{n}\left[i t_{i}^{+}+(i+1 / v) t_{i}^{-}\right] ; \quad Y_{3}=\sum_{i=0}^{n}\left[\left(t_{i}^{+}\right)^{2}+\left(t_{i}^{-}\right)^{2}\right] ; \\
C(\boldsymbol{\theta})=\left(-\frac{\vartheta_{3}}{\pi}\right)^{n+1} \exp \left\{\frac{(n+1) \vartheta_{1}^{2}}{2 \vartheta_{3}}+\frac{(n+1)(n+1 / v) \vartheta_{1} \vartheta_{2}}{2 \vartheta_{3}}+\frac{\vartheta_{2}^{2}}{4 \vartheta_{3}}\left[\frac{n(n+1)(2 n+1)}{6}+\frac{(n+1)(n v+1)}{v^{2}}\right]\right\} .
\end{gathered}
$$

Here the parameter $C(\boldsymbol{\theta})$ is obtained as $C(\boldsymbol{\theta})=\left(-\vartheta_{3} / \pi\right)^{n+1} \exp \left\{C_{1}(\boldsymbol{\theta})+C_{2}(\boldsymbol{\theta})\right\}$, where $C_{1}(\boldsymbol{\theta})$ and $C_{2}(\boldsymbol{\theta})$ are represented by formulas $(20)$ and (21). Thus, the conditional probability density $\rho\left(\mathbf{t}^{+}, \mathbf{t}^{-}\right)$takes the form:

$$
\rho\left(\mathbf{t}^{+}, \mathbf{t}^{-}\right)=h\left(\mathbf{t}^{+}, \mathbf{t}^{-}\right) g\left(\boldsymbol{\theta}, \mathbf{Y}\left(\mathbf{t}^{+}, \mathbf{t}^{-}\right)\right)
$$

where $h\left(\mathbf{t}^{+}, \mathbf{t}^{-}\right) \equiv 1$ and $g(\boldsymbol{\theta}, \mathbf{Y})=C(\boldsymbol{\theta}) \exp \{\mathbf{Y} \cdot \boldsymbol{\theta}\}$ and according to the factorization theorem [28, 32, 33], the statistics $\mathbf{Y}$ expressed by formula (23) are sufficient statistics.

Distribution density $\rho\left(\mathbf{t}^{+}, \mathbf{t}^{-}\right)$belongs to exponential distribution with sufficient statistics $\mathbf{Y}=\left\{Y_{l}, Y_{2}, Y_{3}\right\}$ and parameter $\boldsymbol{\theta}$. For unknowns, $t_{o o}^{+}, T, \sigma_{+}$the parameter takes values from the region $(0, \infty) \times(0, \infty) \times(-\infty, 0)$. We use the completeness theorem [26], according to which if the probability distribution density belongs to the class of exponential distributions and the set of parameter values contains an $n$-dimensional interval, then the statistics $\mathbf{Y}$ is complete [28]. Then we can apply the theorem on the uniqueness of the effective estimate.

Let us express the period in terms of the mathematical expectation of the complete sufficient statistic Y. We calculate the mathematical expectation of $M\left(Y_{l}\right)$ and $M\left(Y_{2}\right)$, and obtain the system of equations for $t_{00}^{+}, T$, v. Having calculated the sums and giving similar ones, we get expressions for $Y_{1}$ and $Y_{2}$ :

$$
\begin{aligned}
& Y_{1}=\sum_{i=0}^{n}\left(\Delta t_{0 i}^{+}+\Delta t_{0 i}^{-}\right)+2(n+1) t_{00}^{+}+(n+1)\left(n+\frac{1}{v}\right) T, \\
& Y_{2}=\sum_{i=0}^{n}\left(\Delta t_{0 i}^{+} i+\Delta t_{0 i}^{-}\left(i+\frac{1}{v}\right)\right)+(n+1)\left(n+\frac{1}{v}\right) t_{00}^{+}+(n+1)\left(\frac{3 n v+v^{2} n(2 n+1)+3}{3 v^{3}}\right) T .
\end{aligned}
$$

Considering that the mathematical expectation of small random additions due to the action of noise with a normal distribution is equal to zero, we obtain the following complete system of linear equations with two unknowns $t_{00}^{+}$and $T$ :

$$
\left\{\begin{array}{l}
M\left(Y_{1}\right)=2(n+1) t_{00}^{+}+(n+1)\left(n+\frac{1}{v}\right) T, \\
M\left(Y_{2}\right)=(n+1)\left(n+\frac{1}{v}\right) t_{00}^{+}+(n+1)\left(\frac{3 n v+v^{2} n(2 n+1)+3}{3 v^{3}}\right) T .
\end{array}\right.
$$

Having solved the system by Cramer's method [31], we obtain the required representation of the period in terms of linear combination of the first moments of the complete statistic Y:

$$
T=\frac{6 v^{2}}{(n+1)\left(n^{2} v^{2}+2 n v^{2}+3\right)}\left[M\left(Y_{2}\right)-\frac{n v+1}{2 v} M\left(Y_{1}\right)\right] .
$$

Thus, the estimate of the pulse repetition period (27) is presented in the form (16). According to the uniqueness theorem for the effective estimate, the only effective estimate of the pulse repetition period is:

$$
\hat{T}=\frac{6 v^{2}}{(n+1)\left(n^{2} v^{2}+2 n v^{2}+3\right)}\left[Y_{2}-\frac{n v+1}{2 v} Y_{1}\right] .
$$

Taking into account the expressions for $Y_{1}$ and $Y_{2}$, the effective estimate of pulses period is: 


$$
\hat{T}=\frac{3 v}{(n+1)\left(n^{2} v^{2}+2 n v^{2}+3\right)} \sum_{i=0}^{n}\left[(2 i v-n v-1) t_{i}^{+}+(2 i v-n v+1) t_{i}^{-}\right]
$$

The variance of the estimate $\hat{T}$ is given by

$$
\begin{gathered}
D(\hat{T})=\frac{(3 v)^{2}}{(n+1)^{2}\left(n^{2} v^{2}+2 n v^{2}+3\right)^{2}} \sum_{i=0}^{n}\left[(2 i v-n v-1)^{2} D\left\{t_{i}^{+}\right\}+(2 i v-n v+1)^{2} D\left\{t_{i}^{-}\right\}\right]= \\
=\frac{(3 v)^{2} \sigma_{+}^{2}}{(n+1)^{2}\left(n^{2} v^{2}+2 n v^{2}+3\right)^{2}} \sum_{i=0}^{n}\left[(2 i v-n v-1)^{2}+(2 i v-n v+1)^{2}\right], \\
D(\hat{T})=\frac{6 v^{2}}{(n+1)\left(n^{2} v^{2}+2 n v^{2}+3\right)} \frac{1}{\left[q s^{\prime}\left(t_{00}^{+}\right)\right]^{2}} .
\end{gathered}
$$

Here we assume that $\mathrm{D}\left\{t_{i}^{+}\right\}=\mathrm{D}\left\{t_{i}^{-}\right\}=\sigma_{+}^{2}=1 /\left[q s^{\prime}\left(t_{00}^{+}\right)\right]^{2}$.

In order to exclude the parameter $s^{\prime}\left(t_{00}^{+}\right)$from the number of a priori unknowns, its estimate in the approximation of a quasirectangular pulse shape is obtained. Based on the criterion of maximum likelihood, the variance $D(\hat{T})$ will be minimal for such value of the threshold $H$, at which the steepness of the signal front at the point of intersection is maximum, i.e. we investigate the derivative of the function $s^{\prime}(x)$ to the maximum absolute value. Therefore, we find the second derivative $s^{\prime \prime}(x)$ (31), equate it to zero and obtain equations (32), (33)

$$
\begin{aligned}
& s^{\prime \prime}(x)= \begin{cases}\frac{\pi k^{2}}{4(k-1)^{4}} \exp \left\{-\frac{\pi}{8} \frac{(2 x k-1)^{2}}{(k-1)^{2}}\right\}\left(4 \pi x^{2} k^{2}-4 \pi x k-4 k^{2}+8 k-4+\pi\right), & x>\frac{1}{2 k}, \\
0, \quad|x| \leq \frac{1}{2 k}, & \\
\frac{\pi k^{2}}{4(k-1)^{4}} \exp \left\{-\frac{\pi}{8} \frac{(2 x k+1)^{2}}{(k-1)^{2}}\right\}\left(4 \pi x^{2} k^{2}+4 \pi x k-4 k^{2}+8 k-4+\pi\right), & x<-\frac{1}{2 k},\end{cases} \\
& 4 \pi x^{2} k^{2}-4 \pi x k-4 k^{2}+8 k-4+\pi=0, \\
& 4 \pi x^{2} k^{2}+4 \pi x k-4 k^{2}+8 k-4+\pi=0 .
\end{aligned}
$$

Each of these equations has two solutions, but we will only consider roots that are greater than $1 / 2 k$ for (32) and less than $-1 / 2 k$ for (33) for $k>1$, since the other roots have no physical meaning. The selected solutions corresponding to equations (32) and (33) have the following form:

$$
\begin{gathered}
x_{00}^{+}=\frac{\pi+2 \sqrt{\pi k^{2}-2 \pi k+\pi}}{2 \pi k}, \\
x_{00}^{-}=\frac{-\pi-2 \sqrt{\pi k^{2}-2 \pi k+\pi}}{2 \pi k} .
\end{gathered}
$$

The derivative of the dimensionless signal $S^{\prime}(x)$ takes the minimum value at $x=x_{00^{+}}$, and the maximum value at $x=x_{00^{-}}$, but in absolute value they are equal and maximum 


$$
\left|s^{\prime}\left(x_{00}^{+}\right)\right|=\left|s^{\prime}\left(x_{00}^{-}\right)\right|=\frac{\sqrt{\pi} k}{\sqrt{e}(k-1)} .
$$

The physical meaning of the obtained variables can be stated as follows: $x 00^{+}-$the initial phase of the quasi-rectangular signal $s(x)$, i.e. the first crossing of the $H$ level by the signal leading edge, at which the variance of the signal period estimate $\hat{T}$ reaches a minimum value; $x 00^{-}$- similarly, only for the trailing edge.

By inverting the variables $t=\tau_{0} x$, we pass to the signal $s(t)$, where $t$ is no longer a dimensionless quantity, but time. Then the desired values $t 00^{+}$and $s^{\prime}\left(t_{00^{+}}\right)$will take the following form:

$$
\begin{gathered}
t_{00}^{+}=\tau_{0} x_{00}^{+}=\tau_{0} \frac{\pi+2 \sqrt{\pi k^{2}-2 \pi k+\pi}}{2 \pi k}, \\
\left|s^{\prime}\left(t_{00}^{+}\right)\right|=\frac{\sqrt{\pi} k}{\tau_{0} \sqrt{e}(k-1)},
\end{gathered}
$$

Taking into account the assumption of the signal off-duty factor $v=\mathrm{T} / \tau_{0}=2$, we presents $\mathrm{s}^{\prime}\left(\mathrm{t}_{00}{ }^{+}\right)$in terms of the period:

$$
\left|s^{\prime}\left(t_{00}^{+}\right)\right|=\frac{2 \sqrt{\pi} k}{T \sqrt{e}(k-1)} .
$$

Substituting the obtained derivative value and the signal off-duty factor value, $v=2$, in (30), for the variance of the signal period estimate, we obtain:

$$
D(\hat{T})=\frac{6}{(n+1)\left(4 n^{2}+8 n+3\right)} \frac{e T^{2}(k-1)^{2}}{q^{2} \pi k^{2}} .
$$

\section{Methods comparison and analysis}

We find the relative error in estimating pulse repetition period obtained by the method of complete sufficient statistics. The relative error for $\mathrm{n}$ measurements is determined as follows:

$$
\delta_{1}=\frac{\sqrt{D(\hat{T})}}{T}=\frac{\sqrt{6 e}}{\sqrt{\pi(n+1)\left(4 n^{2}+8 n+3\right)}} \frac{(k-1)}{q k} .
$$

Consider the relative error $\delta_{1}$, which depends on the signal-to-noise ratio $q$ and the number of measurements $n$, assuming that the squareness coefficient of the pulse $k$ is 1.2 ( $k=1.2$, in practice, may be used a differential scheme to estimate $\mathrm{k}$, which allows to conclude about the steepness of the leading and trailing edges of the pulse):

$$
\delta_{1}=\frac{\sqrt{e}}{q \sqrt{6 \pi(n+1)\left(4 n^{2}+8 n+3\right)}} .
$$

Fig. 2 shows the ratio of the relative errors $\delta 0 / \delta 1$ obtained by the MLE $\delta 0$ and CSS $\delta 1$, calculated using formulas (11) and (42) for a different number of pulses in the sample $n$. The figure shows that this ratio is greater than 1 for $n>8$ and increases with rise in the sample size. This indicates that the estimate obtained by CSS method is more efficient than the MLE estimate for $\mathrm{n}>8$. 


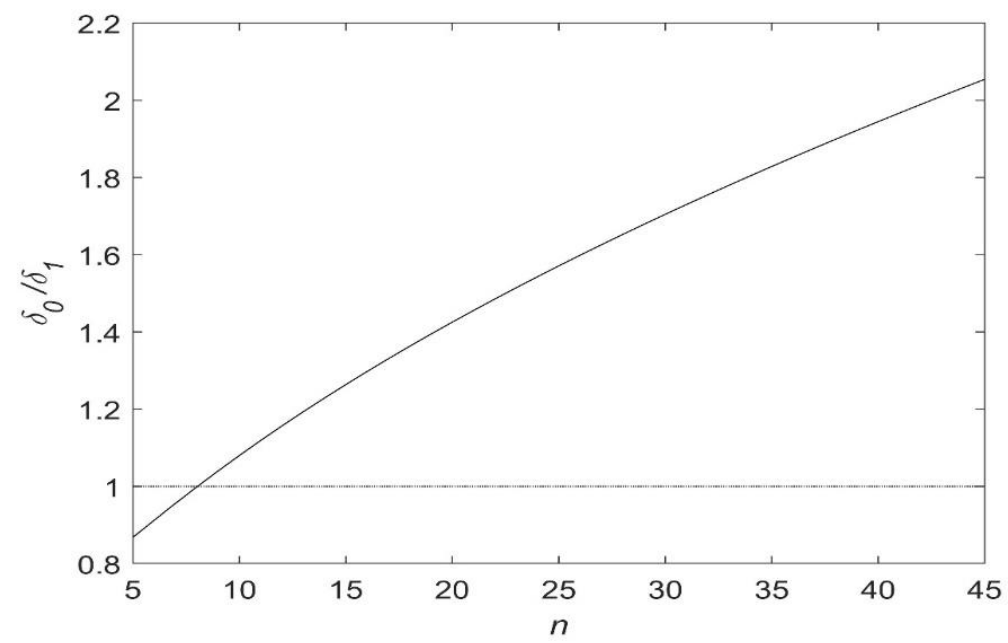

Fig. 2. The ratio of relative pulse period determination errors $\delta 0 / \delta 1$, where $\delta 0$ and $\delta 1$ are relative errors of the MLE and CSS methods respectively.

The greatest gain from using the CSS method can be obtained at low values of the signal-to-noise ratio, when the accuracy of both methods is low. Let us fix the number of measurements (for example, $\mathrm{n}=16$ ) to estimate the energy gain of the CSS methodology in comparison with traditional approaches for the finite and small sample size. The dependences $\delta_{0}$ and $\delta_{1}$ on $q$ in the range of $0-10 \mathrm{~dB}$ will give a difference in the achieved signal-to-noise ratio of the order of $2 \mathrm{~dB}$ in favor of an estimate using the CSS methodology (Fig. 3).

\section{Numerical modeling, results and discussion}

Expressions (11) and (42) only allow us to theoretically estimate the energy gain of the CSS method in comparison with the MLE when solving the problem of estimating the pulse repetition period. At the same time, to verify such estimates, numerical simulations were performed in the MATLAB environment. For this purpose, a sequence of pulses was generated against the background of additive white Gaussian noise with intra-pulse quadrature phase shift keying (QPSK). The order of occurrence of the pulse at a given time position and its carrier frequency in the receiving band were determined by a pseudorandom sequence generator.

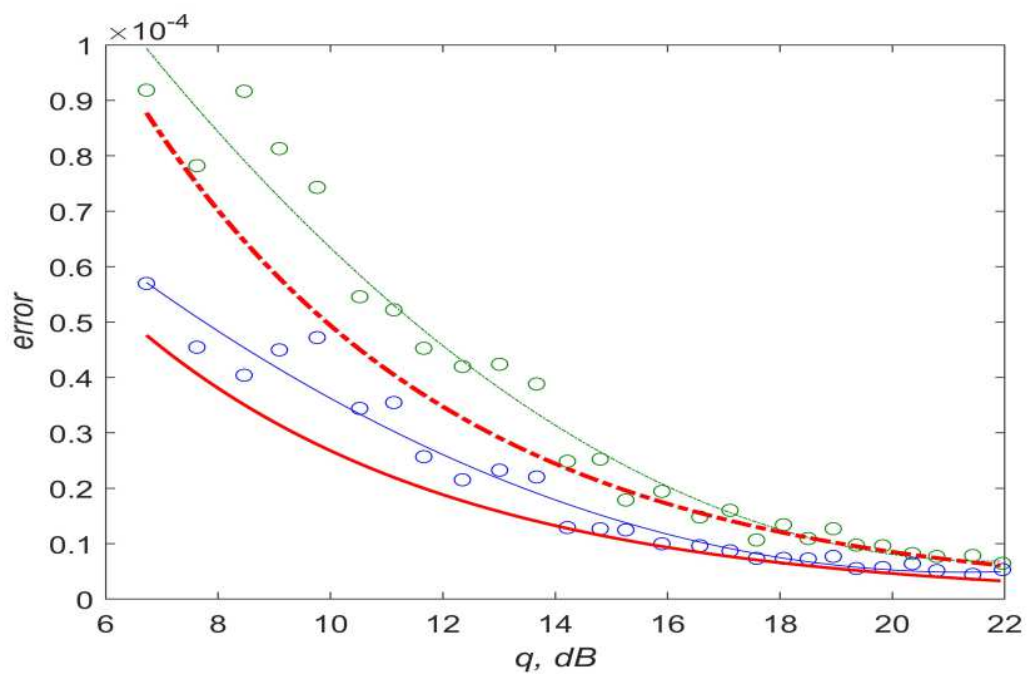

Fig. 3. Relative errors in measuring the pulse repetition period: dotted lines correspond to the MLE, solid lines correspond to the CSS method, bold lines are errors calculated by formulas (11), (42), thin lines are results of least squares method approximations of numerical modeling (circles - results of numerical modeling). The signal-to-noise ratio $(\mathrm{SNR})$ is plotted along the abscissa axis.

Two alternative algorithms for estimating the pulse repetition period were applied to the resulting implementation: based on the MLE and CSS methods, computed according to the formulas (12) and (29). The number of pulses in the implementation $\mathrm{n}$ ranged from 10 to 50 , and the signal-to-noise ratio q ranged from 6 to $22 \mathrm{~dB}$.

The results of the estimation for $\mathrm{n}=16$ as a function of $\mathrm{q}$ are shown in Fig. 3. The relative errors of the two alternative algorithms are presented. It is easy to see that the energy gain of the CSS method is more than $2 \mathrm{~dB}$ in the q interval up to $12 \mathrm{~dB}$. The difference is reduced to $1 \mathrm{~dB}$ with an increase in the signal-to-noise ratio $\mathrm{q}$, while it remains that the traditional estimate asymptotically tends to the unique 
effective one when $q \rightarrow \infty$

In addition, numerical modeling demonstrated the stability of the algorithm work for estimating the pulse repetition period using the CSS methodology with respect to variations in the threshold level H (red line in Fig.1) over a wide range (up to $10 \mathrm{~dB}$ ). With the same variation, the estimate synthesized on the basis of the MLE approach is not stable.

Let us compare the computational costs required to find the pulse repetition period using the MLE and CSS methods. Both methods are linear with respect to the sample size of signal pulses (they belong to the efficiency class $\mathrm{O}(\mathrm{n})$ ). In the case of the traditional estimation of the pulse repetition period, obtained by the MLE method according to formula (12), the number of addition/subtraction $C_{+,-}^{M L E}$, multiplication $C_{*}^{M L E}$ and division $C_{/}^{M L E}$ operations after optimization depends on the number of pulses in the sample as follows

$$
C_{+,-}^{M L E}(n)=3 n+1, C_{*}^{M L E}(n)=0, C_{/}^{M L E}(n)=1+n .
$$

In the case of the pulse repetition period estimation obtained by the CSS method according to formula (27), the number of operations of addition/subtraction $C_{+,-}^{C S S}$, multiplication $C_{*}^{C S S}$ and division $C_{/}^{C S S}$ after optimization is

$$
C_{+,-}^{C S S}(n)=3(n+1)+5, C_{*}^{C S S}(n)=2(n+1)+6, C_{\text {/ }}^{C S S}(n)=1 .
$$

Comparing (43) and (44), we conclude that the CSS method requires more operations, however, additional optimization of expression (29) for a specific processor architecture or logical cell array (LCA) is possible, examples of which are described in [29, 30]. This optimization is a separate engineering problem and is beyond the scope of this work.

\section{Conclusion}

The methodology of complete sufficient statistics is applied to solve the problem of finding an effective estimate of the pulse repetition period. Taking into account the probability distribution functions, a mathematical apparatus is constructed that allows using the LehmannScheffe theorem and its corollary. An analytical expression for the effective estimation of the pulse repetition period is obtained.

Expressions for the variance and relative error of the pulse repetition period estimation are obtained using the CSS methodology depending on the duty-off-factor, squareness coefficient, signal-to-noise ratio and sample size. In the framework of the estimation theory, the lower bound of the accuracy of the estimation of the pulse repetition period is calculated. The relative errors of estimates are compared using traditional methods of MLE and the CSS methodology.

It is shown that for a finite and small value of the sample size $(n=16)$ in the range of signal-to-noise ratios of 5-12 dB, the relative rootmean-square error of estimating the pulse repetition period by CSS method is significantly less than the error of the traditional estimate by MLE method.

The results obtained are applicable for the design of the control and measuring equipment of the oil and gas industry.

\section{Data availability statement.}

The datasets generated during and/or analyzed during the current study are available from the first author on reasonable request.

\section{References}

[1] Abratkiewicz K., Samczyński P., Czarnecki K. 'Radar signal parameters estimation using phase accelerogram in the time-frequency domain' IEEE Sensors Journal. Vol. 19(13), pp. 5078-5085, 2019 doi: 10.1109/JSEN.2019.2903352.

[2] Wu N., Li B., Wang H., Hou L., Kuang J. 'Parametric message passing-based relative navigation in joint tactical information distribution system'. Journal of Systems Engineering and Electronics. Vol. 27. Pp. 81-89, 2016 doi: 10.1109/JSEE.2016.00009.

[3] G. Ravi Shankar Reddy, Rameshwar Rao. 'Oscillatory-plus-transient signal decomposition using TQWT and MCA[j]'. Journal of Electronic Science and Technology, vol. 17(2), pp. 135-151, 2019 doi: 10.11989/JEST.1674-862X.6071911

[4] Matuszewski J. 'The analysis of modern radar signals parameters in Electronic Intelligence system' in Proc. of the 13th International Conference on Modern Problems of Radio Engineering. Telecommunications and Computer Science, TCSET, Ukraine, 2016. Pp. 298-302. doi: 10.1109/TCSET.2016.7452040.

[5] Abe H., Aquilanti G., Boada R., Bunker B., Glatzel P., Nachtegaal M., Pascarelli S. 'Improving the quality of XAFS data'. J. Synchrotron Rad. Vol. 25, pp 972-980, 2018 https://doi.org/10.1107/S1600577518006021

[6] Zhuchkov K. N., Shuvaeva V. A., Yagi K. and Terauchi H.. 'Deglitching procedure for XAFS'. J. Synchrotron Rad. Vol. 8, pp 302-304., 2001 https://doi.org/10.1107/S0909049500020951

[7] Terauchi H., Bobrova N., Sasorov P., Kikuchi T., Sasaki T., Higashiguchi T., Yugami N. and Kodama R. 'Observation and numerical analysis of plasma parameters in a capillary discharge-produced plasma channel waveguide'. Journal of Physics D: Applied Physics, Vol. 109, 053304, 2011. https://doi.org/10.1063/1.3560529

[8] Vasilchenko M., Zavyalov A., Zhuchkov K. 'Increasing the stability of a spatially distributed information system using a robust algorithm for filtering anomalous measurements'. IT in industry, Vol 3(8), pp. 1-7, 2020 https://it-in-industry.com/issue/archive/110.html.

[9] Zhuchkov K.N., Zavyalov A.P., Lukyanov V.A. 'On the necessity to develop approaches to the assessment of reliability parameters of equipment and pipelines of gas transport systems'. Gas industry, Vol. 11 (745), pp. 54-60, 2016 https://elibrary.ru/item.asp?id=27406285

[10] Oparin, V., Vostretsov A., Krivetsky A., Bizyaev A., Yakovitskaya G.. 'Integrated measurement and recording of loads, displacements and electromagnetic emission in rocks under uniaxial compression'. Journal of Mining Science. Vol. 47, pp. 547-557, 2011 doi: 10.1134/S1062739147050017.

[11] Van Trees H. Detection, Estimation, and Modulation Theory, Part I. New York: John Wiley and Sons., 1968 
[12] Levin B. R. Theoretical foundations of statistical radio engineering Vol. 2. Moscow.: Sov. radio, 1975 (in Russian)

[13] Borovkov A. A. Mathematical statistics, Moscow: Nauka, 1984 (in Russian)

[14] Xu L., Ding F. 'Recursive least squares and multi-innovation stochastic gradient parameter estimation methods for signal modeling'. Circuits, Syst. Signal Process, Vol. 36(4), pp. 1735-1753, 2017, doi: 10.1007/s00034-016-0378-4.

[15] Wu M.-W., Jin Y., Li Y., Song T., Kam P.-Y. 'Maximum-likelihood, magnitude-based, amplitude and noise variance estimation'. IEEE Signal Process. Lett., Vol. 28, pp. 414-418, 2021, doi: 10.1109/LSP.2021.3055464.

[16] Li Y., Zhao H., Fan W., Shen C. 'Extended noise resistant correlation method for period estimation of pseudoperiodic signals'. IEEE Trans. Instrum. Meas., Vol. 70, pp. 1-11, 2021, doi: 10.1109/TIM.2021.3052027.

[17] Andrew White, Jung-Wuk Hong, Soonsung Hong, Jongeun Choi 'Parameter estimation for wavelet transformed ultrasonic signals' NDT \& $E$ International. Vol. 44 (1), pp. 32-40, 2011 https://doi.org/10.1016/j.ndteint.2010.09.003

[18] R. Krummenauer, M. Cazarotto, A. Lopes, P. Larzabal, P. Forster 'Improving the threshold performance of maximum likelihood estimation of direction of arrival', Signal Processing, Vol. 90 (5), Pp 1582-1590, 2010, https://doi.org/10.1016/j.sigpro.2009.10.028.

[19] Atamanskiy D.V., Sadovyi K.V. 'Multithreshold Procedure for Evaluating Parameters of Meteorological Objects against the Background of Local Objects Clutter in Pulsed Doppler Weather Radars'. Radioelectron.Commun.Syst. Vol. 61, pp. 317-324, 2018. https://doi.org/10.3103/S073527271807004X

[20] Wright D., Horry R., Skagerberg E.. 'Functions for traditional and multilevel approaches to signal detection theory'. Behavior research methods. Vol. 41. Pp. 257-67, 2009 doi: 10.3758/BRM.41.2.257.

[21] Lo J., Bassu D. 'Adaptive multilayer perceptrons with long- and short-term memories'. IEEE Trans Neural Netw. Vol. 13(1), pp. 22-33, 2002doi: $10.1109 / 72.977262$.

[22] Lehmann E. L., Scheffé H. 'Completeness, similar regions, and unbiased estimation: Part I' Sankhyā: The Indian Journal of Statistics, Vol. 10 (4), pp. 305-340, $1950 \mathrm{https}: / / \mathrm{www} . j$ stor.org/stable/25048038

[23] Parkhomenko N. G., Khoruzhiy S. G., Zhuchkov K. N., Aleksandrov V. P., Stepanov I. M. 'Perspective approaches in signal parameter estimation problems.' Marine Radioelectronics, Vol. 16 (2), pp. 46-48, 2006 http://mr.shipbuilding.ru/magazine/n16/.

[24] Kay S.M. Fundamentals of Statistical Signal Processing: Estimation Theory. - NJ.: Prentice Hall PTR, 1985.

[25] Krasnov A. Yu., Zhuchkov K. N., Khoruzhiy S. G. 'Determination of the initial phase of the clock oscillation for pulse signals with MSK'. in Proc. of the 7th International conference on digital signal processing and its application, DSPA, Moscow, Russia, 2005, pp. 192-195 http://www.autex.spb.su/download/dsp/dspa/dspa2005/t1/16.pdf.

[26] Leonov G.A., Kuznetsov, N.V., Yuldashev M.V., Yuldashev R.V. 'Hold-in, pull-in, and lock-in ranges of PLL circuits: rigorous mathematical definitions and limitations of classical theory'. Circuits and Systems I: IEEE Transactions. Vol. 62 (10) Pp. 2454-2464., 2015 doi:10.1109/TCSI.2015.2476295.

[27] Trifonov A. P., Bespalova M. B. ,'Quasi-plausible estimation of the arrival time and period of video pulses’ Radiotechnika, vol. 3 pp. 78-80, 1998 https://phys.vsu.ru/radio/sites/default/files/publfile/174_radiotehnika_1998-3.pdf

[28] Bogdanovich V.A., Vostretsov A.G. The theory of robust detection, discrimination and estimation of signals. - Moscow: Physmathlit, 2004. (in Russian)

[29] Govorukhina A., Zhuchkov K., Khoruzhiy S. 'Code optimization methods for the TMS320C6000 Digital Signal Processor'. Digital signal processing. Vol.4. pp. 47-56, 2004 http://www.dspa.ru/articles/year2004/jour04 4/art04 4 8.djvu

[30] Wallace R, Marvuglia A., Benetto E., Tiruta-Barna L. 'A new approach to optimization with life cycle assessment: combining optimization with detailed process simulation' In: Murgante B. et al. (eds) Computational Science and Its Applications - ICCSA 2014. Lecture Notes in Computer Science, vol 8581. Springer, Cham. https://doi.org/10.1007/978-3-319-09150-1 52

[31] Cramer G. 'Introduction à l'Analyse des lignes Courbes algébriques ‘.- Geneva: Europeana (1750). (in French)

[32] Fisher-Neyman factorization theorem [Electronic resource]: Wikipedia. Free encyclopedia. URL: https://en.wikipedia.org/wiki/Sufficient_statistic\#Fisher\%E2\%80\%93Neyman factorization theorem

[33] Lehmann E.L., Casella G. Theory of point estimation. - New York: Springer Science \& Business Media, 2006,560 p.

[34] Lyne, A., Rickett, B. Measurements of the Pulse Shape and Spectra of the Pulsating Radio Sources. Nature 218,pp. 326-330,1968. https://doi.org/10.1038/218326a0

[35] Morel, B., Giust, R., Ardaneh, K. et al. A solver based on pseudo-spectral analytical time-domain method for the two-fluid plasma model. Sci Rep 11, pp. 3151, 2021. https://doi.org/10.1038/s41598-021-82173-9 